\section{Presoaking with BSS used for thin manually dissected DSEK (TMDSEK): a viable option for thin DSEK}

M Tsatsos ${ }^{1}$, A Konstantopoulos ${ }^{1,2}$, P Hossain ${ }^{1,2}$ and $\mathrm{D}$ Anderson ${ }^{1}$

\begin{abstract}
Aim To describe a novel technique for the safe manual dissection of thin donor lenticules in $\mathbf{1 0}$ consecutive patients undergoing DSEK surgery. Methods A key element of our new technique was to presoak the donor cornea in balanced salt solution (BSS) for $30 \mathrm{~min}$ before manual dissection. The cornea was placed on an artificial anterior chamber and pressure in the chamber was maintained at $80 \mathrm{~mm} \mathrm{Hg}$. The limbus of the donor cornea was incised to the same depth as the central corneal thickness. Lamellar dissection was started with the short side of the Morlet dissector (Duckworth \& Kent

\section{Introduction}

Increasingly, corneal surgeons have adopted DSEK as the surgical treatment of choice for patients with endothelial disease. Although there is debate as to whether the thickness of the donor corneal lenticule is related to visual outcome, there is a growing view that thinner donor lenticules result in superior visual outcomes.

A variety of techniques for preparation of the donor lenticule, such as automated ${ }^{1-3}$ or manual ${ }^{4}$ corneal dissection, have been described which generally generate donor lenticules of $150 \mu \mathrm{m}$ or more.
\end{abstract} Ltd) and completed using the lamellar (less sharp) end of the Morlet dissector. Outcomes of $\mathbf{1 0}$ consecutive cases of thin manually dissected DSEK (TMDSEK) were prospectively analysed for thickness and visual outcome.

Results Mean graft thicknesses measured less than $100 \mu \mathrm{m}$ at 1 month post surgery (mean thickness $90.7 \mu \mathrm{m}$, range $48-137 \mu \mathrm{m}$, SD $29.96 \mu \mathrm{m})$. Presoaked donor corneal pachymetry was strongly negatively correlated with graft thickness (correlation $r=-0.75, P<0.05)$.

Discussion Our dissection technique achieves consistently thin endothelial donor corneal grafts that can be safely produced with minimal financial investment and no limitations on surgical time. This technique is likely to be of significant importance for a large proportion of the eye centres where microkeratomes may not be routinely available. Eye (2014) 28, 701-704; doi:10.1038/eye.2014.36; published online 14 March 2014

\section{Aims}

We present 10 consecutive cases using our technique, thin manually dissected DSEK (TMDSEK), for the safe and consistent creation of thin donor corneal lenticules.

Materials and methods

Patients

Patients undergoing TMDSEK at University Hospital Southampton National Health Service (NHS) Foundation Trust over a 7-month period were prospectively recruited. Local Ethics Committee and National Health Service Research and Development approval was obtained (Research Ethics Committee number: 07/H0512/39).

Ten consecutive eyes of 10 patients were used. All 10 cases were included in the keratometric analysis. All patients included in the study were pseudophakic. Three cases were excluded from
${ }^{1}$ Department of Ophthalmology, Cornea and External Disease Service, Southampton Eye Unit, Southampton University Hospitals NHS Trust, Southampton, UK

${ }^{2}$ Division of Infection, Inflammation and Immunity, Faculty of Medicine, University of Southampton, Southampton, UK

Correspondence: M Tsatsos, University of Southampton, Eye Unit, MP104, Southampton General Hospital, Tremona Road, Southampton SO16 6YD, UK.

Tel: + 44238079 4579;

Fax: +44 2380794120 .

E-mail: michaeltsatsos@ hotmail.com

Received: 7 March 2013 Accepted in revised form: 29 January 2014 Published online: 14 March 2014 
best corrected visual acuity (BCVA) analysis due to reduced visual potential (visual improvement limited due to comorbidity-significant ARMD). Indications for TMDSEK were Fuchs' endothelial dystrophy $(n=8)$ and pseudophakic bullous keratopathy $(n=2)$. Hand movements and perception of light were assigned the values of 1.9 and $2.2 \log$ MAR, respectively. Normality of data was confirmed using the Shapiro-Wilk test and the package used for statistical analysis was SPSS v19 (IBM SPSS, Chicago, IL, USA).

\section{Technique-graft dissection}

All donor corneas were supplied by the NHS BT (National Health Service Blood Transfusion) in transport medium containing dextran and were placed in BSS (Alcon Labs, Fort Worth, TX, USA) for $30 \mathrm{~min}$ immediately before surgery. A small amount of viscoelastic (Healon; AMO, Santa Ana, CA, USA) was placed in the centre and on the edges of the base of the artificial anterior chamber (AC) (Baron artificial anterior chamber; Katena Products, Denville, NJ, USA) to protect the donor corneal endothelial cells. The pressure inside the artificial AC was maintained at $80 \mathrm{~mm} \mathrm{Hg}$ with BSS. Ten ultrasound pachymetry (Tomey, SP-3000; Tomey Corporation, Nagoya, Japan) measurements of the central corneal thickness (CCT) were taken and their average was noted.

The limbus of donor cornea was incised with a guarded diamond knife (Duckworth \& Kent Ltd, Baldock, UK) set to the same depth as the mean (CCT) measurement. The incision was started at the side of the cornea where arcus senilis (where present) was most prominent. The short side of Morlet dissector (Duckworth \& Kent Ltd) was placed deep in the incision to ensure a dissection at the calculated depth

(Figure 1). The dissection was then extended across the cornea using the lamellar (less sharp) end of the Morlet dissector so as to maintain the same plane of dissection with minimal risk of creating a new passage each time a fresh part of the cornea was dissected (see Supplementary video).

The rest of the DSEK surgery was performed as previously described ${ }^{5}$ with an injectable device (The Endosaver, Ocular Systems, Inc., Winston-Salem, NC, USA) through a $4.5-\mathrm{mm}$ incision. ${ }^{5}$

\section{Postoperative care}

All patients were asked to remain supine for $2 \mathrm{~h}$ and intra-ocular pressure was measured $4 \mathrm{~h}$ postoperatively during slit-lamp examination.

\section{Results}

All 10 cases were successfully dissected with no perioperative complications, such as lenticule perforation. Diagnosis, preoperative and postoperative BCVA, pachymetry, and endothelial cell counts are summarized in Table 1. Mean BCVA was $0.3 \log$ MAR 1 month post TMDSEK (range 0.2-0.4), significantly better $(P<0.05$ $t$-test) than preoperative mean 0.8 logMAR (range 0.5-2.2). Grafts 3, 4, and 6 were excluded from the vision analysis due to reduced visual potential related to ARMD although they led to a substantial improvement of vision. Graft number 3 was performed for pseudophakic bullous keratopathy to alleviate pain and discomfort. In the other two cases (graft 4 and 6) the patients were very keen to proceed with DSEK in an attempt to improve their overall visual acuity.

Mean donor acoustic pachymetry performed on the operating table before initiation of the corneal dissection was $593.3 \mu \mathrm{m}$ (range 514-730, SD 73.25). Mean donor graft thickness measured 1 month postoperatively was $90.7 \mu \mathrm{m}$ (range 48-137 $\mu \mathrm{m}$, SD $29.96 \mu \mathrm{m}$ ). Postsoaking pachymetry ranged between 514 and $730 \mathrm{~mm}$ and was negatively correlated with graft thickness 1 month postoperatively (correlation $r=-0.75, P<0.05$ ). Mean total corneal thickness was $609.9 \mu \mathrm{m}$ (range 504-647 $\mu \mathrm{m}$, SD 48.77) 1 month post TMDSEK. There was no significant correlation between the endothelial count and the corneal thickness of the donor after presoaking $(r=-0.327, P>0.05)$. Mean dissection time was $6.8 \mathrm{~min}$ (range 5-10.5).

\section{Conclusion}

We report 10 consecutive cases of our novel technique for the manual dissection of consistently thin DSEK donor lenticules. There were no dissection complications and the lenticules were consistently thin (SD $29.96 \mu \mathrm{m}$ ). We found a significant correlation between donor corneal thickness (after presoaking) and DSEK lenticule thickness with thicker donor corneas associated with thinner DSEK grafts.

A key element of this method was the presoaking of the donor corneal tissue for 30 min before manual graft dissection that was not part of our old manual technique. We believe that the artificially induced oedema of the donor cornea leads to a smooth and safe dissection. The swollen corneal tissues show reduced resistance during the passage of the Morlet dissector during lamellar dissection, contributing to the safety (no perforations) and speed (mean dissection time of $6.8 \mathrm{~min}$ ) of the procedure. A temporarily thicker donor lenticule also allowed easier intra-operative manipulation with minimal detrimental effect to the endothelium. 
Before the alteration of our technique, the donor cornea was removed from the transport medium and placed directly onto the artificial AC without presoaking in BSS. This did not produce the temporary corneal swelling used in TMDSEK that allows the surgeon to reach deeper dissection planes with minimal resistance.

Our 1 month postoperative mean graft thickness was shown to be $90.7 \mu \mathrm{m}$ compared with a median of $142 \mu \mathrm{m}$ (mean 156 at 1 month) in our previously published cohort. ${ }^{5}$ The variation in thickness was small, showing that this technique can produce consistent results. Vision with TMDSEK was significantly better even at 1 month following surgery, despite the small number of cases. Mean BCVA at 1 month post DSEK (with our old technique) was a median of $0.57 \log$ MAR and a mean of $0.42 \log$ MAR. Our initial cohort of 61 patients included 3 donor corneal perforations. We postulate that this difference in the number of perforations can be attributed to the improved new technique, where presoaking provided a reduced level of resistance during dissection.

However, the increasing expertise with dissection and the smaller number of cases may also account for this.

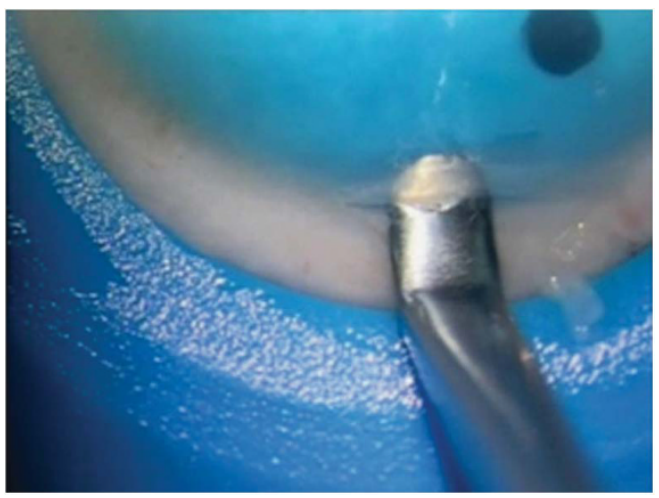

Figure 1 Initiation of the corneal dissection with the sharp end of the Morlet dissector.
The expanded corneal tissues after $30 \mathrm{~min}$ of presoaking in BSS enable a thin and smooth (see Figure 2 and Supplementary video) dissection without the need for a second dissection ${ }^{6}$ leaving the anterior lamellar intact for potential use in anterior lamellar surgery. A mean dissection time of $6.8 \mathrm{~min}$ shows that manual dissection of thin DSEK grafts can be safely performed during a period of time that is not largely different to the time needed for an automated dissection (with the use of a microkeratome).

In this cohort 3 cases, numbers 2, 3, and 7 appeared to have a CCT less than what is considered as physiological even after presoaking with BSS. We acknowledge that these cases were not as swollen as the rest of the cohort; however, an important detail that needs to be stressed is that corneal grafts in the United Kingdom are sent to ophthalmic transplant units in transport medium containing dextran, which is a thinning medium. Although, we did not measure the donor corneal thickness before but only after soaking in BSS to avoid excessive manipulation, the fact that even these three cases were close to a physiological thickness implies that some swelling did occur, albeit less than in the rest of the cohort. We can only speculate that corneas which had a lower baseline (before soaking in BSS) thickness would expand less compared with thicker corneas at baseline (before soaking). These differences in corneal thickness are expected to be exacerbated after soaking in BSS for $30 \mathrm{~min}$.

This 10 case cohort serves to describe our new manual technique for the dissection of thin DSEK donor

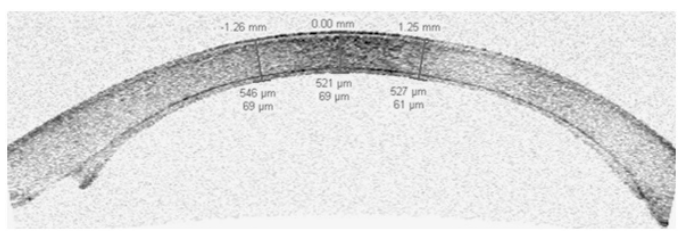

Figure 2 Thin manually dissected DSEK showing the smooth dissection.

Table 1 Showing the indication for DSEK, preop and postop best corrected visual acuity, endothelial counts, postsoaking corneal thickness, postop donor and total corneal thickness

\begin{tabular}{llcccccc}
\hline Case Diagnosis & $\begin{array}{c}\text { Preop BCVA } \\
(\log M A R)\end{array}$ & $\begin{array}{c}\text { Postop BCVA } \\
(\text { logMAR) }\end{array}$ & $\begin{array}{c}\text { Endothelial count } \\
(\text { donor })\end{array}$ & $\begin{array}{c}\text { Postsoaking } \\
\text { pachymetry }(\mu \mathrm{m})\end{array}$ & $\begin{array}{c}\text { Postop donor } \\
\text { lenticule }(\mu \mathrm{m})\end{array}$ & $\begin{array}{c}\text { Postop total corneal } \\
\text { thickness }(\mu \mathrm{m})\end{array}$ \\
\hline 1 & PBK & 0.5 & 0.3 & 2600 & 670 & 50 & 647 \\
2 & Fuchs & 3 & 0.4 & 2600 & 514 & 117 & 635 \\
3 & PBK & 0.9 & 0.8 & 2600 & 514 & 122 & 76 \\
4 & Fuchs & 1.9 & 0.7 & 2800 & 570 & 137 & 636 \\
5 & Fuchs & 0.7 & 0.4 & 2800 & 560 & 69 & 628 \\
6 & Fuchs & 0.9 & 0.6 & 2700 & 598 & 97 & 590 \\
7 & Fuchs & 0.5 & 0.3 & 2700 & 520 & 48 & 634 \\
8 & Fuchs & 0.7 & 0.3 & 2600 & 630 & 98 & 504 \\
9 & Fuchs & 1.5 & 0.4 & 2600 & 600 & 93 & 623 \\
10 & Fuchs & 0.7 & 0.2 & 2600 & &
\end{tabular}


lenticules. Although, larger studies and duplication of this technique are needed, we believe that useful points and concepts can be drawn from our study. The key element of presoaking that leads to the expansion of the donor stroma facilitates the procedure. We believe that artificially induced oedema of the donor cornea leads to a smooth and safe dissection. A thicker donor lenticule allows easier intra-operative manipulation and once inside the eye and attached to the host corneal stroma donor lenticule thickness quickly reduces to reach a mean of $90.7 \mu \mathrm{m} 1$ month postoperatively with good visual recovery.

We present our technique of manual dissection of the graft lenticule for endothelial keratoplasty, TMDSEK. This is a novel technique that uses corneal presoaking in BSS to facilitate dissection and enable easier manipulation of the donor lenticule. Consistently thin donor lenticules were prepared with no complications. A second dissection is not used leaving the anterior stromal available for potential use in anterior lamellar surgery. While DSAEK may offer the gold standard for the creation of thin endothelial grafts, ${ }^{6,7}$ we believe that the TMDSEK technique offers a viable alternative for eye units where keratomes are not routinely available. ${ }^{8}$

\section{Summary}

What was known before

- Manually dissected DSEK grafts are generally thicker than DSAEK

- Manual dissection of donor corneas for DSEK normally leads to higher rate of perforation compared with DSAEK.

- Manual DSEK dissection normally has a much higher duration compared with DSAEK and is technically harder.

What this study adds

- Presoaking with balanced salt solution (BSS) leads to a safer and thinner dissection.

- There is no compromise in terms of surgical time.

- A smooth manual dissection would be of benefit for a large number of eye units worldwide with no access to a keratome and it would allow use of the anterior lamella for anterior lamellar surgery.

\section{Conflict of interest}

The authors declare no conflict of interest.

\section{Author contributions}

MT: substantial contribution to concept and design, acquisition of data, analysis and interpretation of data; drafting and critical review of the manuscript; final approval of the manuscript. AK and $\mathrm{PH}$ : drafting the article, viewing it critically for intellectual content and final approval of the article. DA: drafting the manuscript, viewing it critically for important intellectual content and final approval of the article.

\section{References}

1 Khor WB, Mehta JS, Tan DT. Descemet stripping automated endothelial keratoplasty with a graft insertion device: surgical technique and early clinical results. Am J Ophthalmol 2011; 151(2): 223-232.

2 Sikder S, Nordgren RN, Neravetla SR, Moshirfar M. Ultra-thin donor tissue preparation for endothelial keratoplasty with a double-pass microkeratome. Am J Ophthalmol 2011; 152(2): 202-208.

3 Foster JB, Swan KR, Vasan RA, Greven MA, Walter KA. Small-incision descemet stripping automated endothelial keratoplasty: a comparison of small-incision tissue injector and forceps techniques. Cornea 2012; 31(1): $42-47$.

4 Rice A, Spokes DM, Anand S, Ball JL. Endothelial cell survival and graft profile analysis in descemet stripping endothelial keratoplasty. Cornea 2011; 30(8): 865-871.

5 Shinton AJ, Tsatsos M, Konstantopoulos A, Goverdhan S, Elsahn AF, Anderson DF et al. Impact of graft thickness on visual acuity after Descemet's stripping endothelial keratoplasty. Br J Ophthalmol 2012; 96(2): 246-249.

6 Busin M, Patel AK, Scorcia V, Ponzin D. Microkeratomeassisted preparation of ultrathin grafts for descemet stripping automated endothelial keratoplasty. Invest Ophthalmol Vis Sci 2012; 53(1): 521-524.

7 Price Jr FW, Price MO. Descemet's stripping with endothelial keratoplasty in 50 eyes: a refractive neutral corneal transplant. J Refract Surg 2005; 21: 339-345.

8 Neff KD, Biber JM, Holland EJ. Comparison of central corneal graft thickness to visual acuity outcomes in endothelial keratoplasty. Cornea 2011; 30(4): 388-391.

Supplementary Information accompanies this paper on Eye website (http://www.nature.com/eye) 